University of South Carolina

Scholar Commons

$2-2007$

\title{
Validation of the Physical Activity Questionnaire for Older Children in Children of Different Races
}

\author{
Justin B. Moore \\ University of South Carolina - Columbia, jusmoore@wakehealth.edu \\ John C. Hanes Jr. \\ Paule Barbeau \\ Bernard Gutin \\ Roberto P. Treviño
}

See next page for additional authors

Follow this and additional works at: https://scholarcommons.sc.edu/

sph_health_promotion_education_behavior_facpub

Part of the Public Health Commons

\section{Publication Info}

Published in Pediatric Exercise Science, Volume 19, Issue 1, 2007, pages 6-19.

Moore, J. B., Hanes, Jr., J. C., Barbeau, P., Gutin, B., Treviño, R. P., \& Yin, Z. (2007). Validation of the Physical Activity Questionnaire for Older Children in children of different races. Pediatric Exercise Science, 19(1), 6-19.

(C) Pediatric Exercise Science, 2007, Human Kinetics

This Article is brought to you by the Health Promotion, Education, and Behavior at Scholar Commons. It has been accepted for inclusion in Faculty Publications by an authorized administrator of Scholar Commons. For more information, please contact digres@mailbox.sc.edu. 


\section{Author(s)}

Justin B. Moore, John C. Hanes Jr., Paule Barbeau, Bernard Gutin, Roberto P. Treviño, and Zenong Yin 
ORIGINAL RESEARCH

Pediatric Exercise Science, 2007, 19, 6-19

(C) 2007 Human Kinetics, Inc.

\title{
Validation of the Physical Activity Questionnaire for Older Children in Children of Different Races
}

\author{
Justin B. Moore, John C. Hanes, Jr., Paule Barbeau, \\ Bernard Gutin, Roberto P. Treviño, and Zenong Yin
}

\begin{abstract}
The Physical Activity Questionnaire for Older Children (PAQ-C) is a validated self-report measure of physical activity widely used to assess physical activity in children (8-14 years of age). To date, however, the instrument has been validated in largely White Canadian samples. The purpose of the present article is to determine the psychometric properties of the PAQ-C for African American, European American, and Hispanic children. Two studies were conducted in which independent samples were administered the PAQ-C, along with varying indices of cardiovascular fitness, fatness, and psychological measures related to physical activity. Results showed that the reliability and validity of the PAQ-C varied by race and that modifications might be necessary.
\end{abstract}

Key Words: measurement, youth, racial differences, psychometrics

Physical activity has been identified as a leading health indicator in Healthy People 2010, and recommendations have been issued by professional bodies for a minimal level of physical activity for children (27) and adolescents (31). A recent report from the Centers for Disease Control and Prevention indicated that only 38\% of children ages 9-13 years reported participation in organized physical activity and that only $77 \%$ reported any leisure-time physical activity. During the same period of time, however, 33\% of adolescents (Grades 9-12) reported insufficient levels of moderate and vigorous physical activity (6).

Inactivity in youths is related to adiposity (11), which in turn is related to risk factors for cardiovascular disease (24). Regular physical activity is inversely related to the percentage of body fat and levels of lipids and triglycerides $(33,36)$ and positively related to bone-mineral density $(2,41)$ and cardiovascular fitness

Moore is with the Division of Community Health and Preventive Medicine, The Brody School of Medicine at East Carolina University, Greenville, NC 27834. Hanes is with the School of Education, Regent University, Virginia Beach, VA 23464. Barbeau and Gutin are with the Dept. of Pediatrics, Medical College of Georgia, Augusta, GA 30912. Treviño is with the Social \& Health Research Center, 1302 So. St. Mary's St., San Antonio, TX 78205. Yin is with the Dept. of Health and Kinesiology, University of Texas at San Antonio, San Antonio, TX 78249. 
$(15,28)$ in youths $(15-17$ years of age). In addition to physiological benefit, physical activity is positively related to self-esteem $(35,38)$ and negatively related to depression $(26,37)$ in children and adolescents. It is for this reason that promotion of physical activity is vital to children and adolescents.

In order to determine levels of physical activity in different populations, accurate measurement tools are needed, although many previous studies were based on self-reported physical activity (34). Although accuracy in measurement is of the utmost importance, the most accurate measures of physical activity are often invasive or expensive to use in large populations. For example, direct calorimetry or direct observation is accurate but invasive, and accelerometry and the use of doubly-labeled water is accurate but expensive. Therefore, many studies that measure physical activity in children rely on self-reports, which often have poor psychometric properties or lack sufficient validation (21). Most large studies rely on self-reports of physical activity, which for children and adolescents have questionable validity (31). Furthermore, many measurement tools used for adolescents cannot be used with younger children, thus making longitudinal studies or cross-sectional comparisons difficult.

One potentially valuable instrument for use with children and adolescents is the Physical Activity Questionnaire for Older Children (PAQ-C) (7). The PAQ-C is a 7-day guided, self-administered questionnaire designed to measure moderate to vigorous physical activity (MVPA) in children ages 9-15 years. The PAQ-C has demonstrated good internal consistency; test-retest reliability; and has been shown to be correlated with athletic competence $(r=.48)$, teachers' rating of physical activity $(r=.45)$, fitness assessed via a step test $(r=.28)$, and physical activity measured by accelerometry $(r=.39)(21)$. In longitudinal studies the PAQ-C offers comparability to the Physical Activity Questionnaire for Adolescents (PAQ-A), an instrument that is identical to the PAQ-C except for the omission of a question concerning activity at recess. The PAQ-C, however, has been exclusively used in study samples who were predominantly of European ancestry in Canada. Therefore, further validation of the PAQ-C is valuable for researchers in need of instrumentation to discern activity levels across a wide age range in youths.

The promotion of physical activity is important for all children, but it is especially important for African American and Hispanic children who engage in less physical activity than their European American counterparts $(32,40)$. This trend appears to begin in late childhood, with greater differences seen in adolescence $(19,29)$, and continues into adulthood $(3,5,40)$. Although there is a dearth of instruments for the measurement of physical activity in children, there are even fewer instruments that have been validated in minority youth populations.

This article presents the reliability and validity of the PAQ-C in children and adolescents of different races in two studies. Study 1 was designed to determine the factor structure of the PAQ-C in a predominantly Hispanic sample using exploratory-factor analysis (EFA) and confirmatory-factor analysis (CFA). Furthermore, Study 1 sought to determine the construct validity of the PAQ-C. Study 2 was designed to confirm the factor structure in a population of African American and European American children, as well as to establish the concurrent and construct validity of the PAQ-C. 


\section{Study 1}

\section{Method}

Participants and Procedures. Participants for Study $1(N=1,172)$ were part of a clinical trial conducted by the Social and Health Research Center (The Bienestar Health Program) in a large urban school district in the southwestern United States (39). Self-reported race for the participants was $81 \%$ Hispanic, 9.6\% African American, 4.9\% European American, and 4.4\% "other." For the purpose of statistical analysis, this study included participants for whom complete data were available and who indicated that they were not sick or prevented from engaging in regular physical activity (Question 10 of the PAQ-C). Therefore, the present study only used data from 991 students (mean age $=10.74 \pm 0.49$ years; 808 Hispanic, 51 European American, 91 African American, 41 other; 494 male, 490 female, and 7 not indicating gender). To determine the validity of the implied single-factor structure (7), the sample was split using the SPSS 11.5 SPLIT function (SPSS, Inc., Chicago, IL), which produced two approximately equal samples. All procedures were approved by the Internal Review Board at the University of Texas Health Science Center at San Antonio. The informed written consent of the parent or legal guardian of each participant was obtained, along with the assent of the participants, before data collection.

Measures. Physical activity was assessed using the PAQ-C (7). The PAQ-C is a nine-item, 7-day physical-activity recall designed for use with elementary- and middle-school children in a field-based setting. A tenth item not used in calculation of the activity score asks children if they were sick or otherwise prevented from engaging in regular physical activity. The PAQ-C is designed to be administered once and asks children to recall their participation in activities over the last 7 days to compute an activity score, but it is not intended to estimate metabolic-equivalent expenditure. The PAQ-C has been shown to be internally consistent in children 9-15 years of age, with a Cronbach's alpha of .79-.89 reported (7). The mean of all items is used to indicate level of physical activity. A high score indicates higher levels of physical activity. Details to score the PAQ-C are provided elsewhere (7).

Cardiovascular fitness (CVF) for Study 1 was assessed using a modified version of the Harvard step test. The step-test protocol required the participant to step up and down on a $30-\mathrm{cm}(12-\mathrm{in}$.) bench at a rate of $30 \mathrm{steps} / \mathrm{min}$ for $5 \mathrm{~min}$. Heart rate was recorded using a Polar heart-rate monitor (Polar Vantage XL, Polar Electric Company, Port Washington, NY) immediately on cessation of the test $\left(t_{0}\right)$ and at 1 $\left(t_{1}\right)$ and $2 \min \left(t_{2}\right)$ posttest. If the test was prematurely stopped by the child or by the research assistant because of contraindications to continuation, time at cessation was recorded. A CVF score was calculated by taking the total exercise time in seconds $\left(t_{\mathrm{s}}\right)$ multiplied by 100 and then divided by the sum of the three heart-rate values measured postexercise: $\mathrm{CVF}=\left(t_{\mathrm{s}} \times 100\right) /\left(t_{0}+t_{1}+t_{2}\right)$.

Weight and height were recorded using a digital scale (Tanita Corp. of America, Inc., Skokie, IL) and a Seca Wall Stop measuring tape (Seca Corporation, Hanover, MD). Both height (accurate to $0.1 \mathrm{~cm}$ ) and weight (accurate to $0.5 \mathrm{~kg}$ ) were recorded twice and averaged to produce the final value. Body-mass index (BMI) was calculated as weight in kilograms divided by the square of the standing height in meters 
squared: $\mathrm{BMI}=\left(\mathrm{kg} / \mathrm{m}^{2}\right)$. Height and weight were measured without shoes and in the clothes worn to school. Body-fat percentage was measured using bioelectricimpedance analysis (BIA; Tanita Corp. of America). The bioelectric-impedanceanalysis system consists of a laptop computer and stainless-steel electrode box. For body composition and fasting glucose testing, participants were required to fast overnight and asked to refrain from drinking any fluids before testing. Immediately before testing, participants were asked to remove their shoes and socks and stand on the electrode box. The bioelectric-impedance-analysis system produces the participant's measurement within $30 \mathrm{~s}$. Bioelectric-impedance analysis was used for the present study because of its ease of transport, low expense, and acceptable validity (12).

Fasting capillary glucose was obtained in the morning before the measurement of the other variables. For the testing, participants were required to fast overnight. Research assistants contacted the parents of the participants the evening before data collection to remind them of the fasting requirement. Blood was obtained through a single finger stick on the participant's nondominant hand. Breakfast was given to the participant after data collection. The blood was analyzed with a Glucometer Elite XL (Bayer Corp., Mishawaka, IN), which has been shown to provide a valid, field-based glucose measure (13). If a participant's fasting capillary glucose was greater than $110 \mathrm{mg} / \mathrm{dL}$, a written notice was sent to the parent or guardian and a physician referral was given. This study was conducted before the new American Diabetes Association guidelines for diagnosing diabetes were published (1).

\section{Statistical Analysis}

An analysis of covariance (ANOVA) was conducted to determine any race or gender differences on the PAQ-C while controlling for the effect of age. To determine the concurrent validity of the PAQ-C, Pearson product-moment correlations were calculated for all measured variables. The entire sample was used for the construct-validity analysis because of the low numbers of non-Hispanic children. SPSS 11.05 was used to produce these statistics.

An exploratory-factor analysis employing a principal-axis-factoring extraction with a varimax (orthogonal) rotation was performed for Sample 1. Confirmatoryfactor analysis (CFA) was conducted on Sample 2 to test the structure of the PAQ$\mathrm{C}$ based on the results of the exploratory-factor analysis using Sample 1. Model estimation was performed by a weighted least-squares method using a polychoric correlation matrix weighted by its asymptotic covariance because of the use of the ordinal scoring scale in the PAQ-C. CFA was performed using the measurement submodels of LISREL 8.72 (18).

The appropriateness of the model was assessed by using several indices of fit because each provided a different perspective of model estimation. These included the chi-square statistic, the comparative-fit index (CFI), the nonnormed-fit index (NNFI), and the root-mean-square error of approximation (RMSEA). All four have been widely used in the general literature of analysis of covariance (20), as well as in the literature of exercise psychology $(8,25)$. The criterion for goodness of fit was a small chi-square statistic relative to degrees of freedom resulting in a significant $p$ statistic. It is well known that the chi-square statistic is sensitive to a number of sample factors (including sample size and complexity), but the CFI 
and NNFI have been demonstrated to have strong resistance to the influence of sample size (20). The RMSEA represents the closeness of fit and adjusts for degrees of freedom (22). An RMSEA value below .08 was considered acceptable model fit, below .05 was considered close fit, and a value exceeding .10 was considered unacceptable (4). The adequacy of parameter estimates was assessed by $t$ value ( $t=$ [unstandardized estimate]/[standard error]). A $t$ value greater than 1.96 was considered indicative of good fit.

\section{Results}

Descriptive Statistics (Samples 1 and 2). Descriptive statistics for each of the samples are listed in Table 1. After we adjusted for age, there was no significant sex effect or interaction effect between sex and age or race and age for PAQ-C scores. The internal-consistency coefficient (Cronbach's $\alpha$ ) was .74 for Sample 1 and .70 for Sample 2. Corrected item-total correlations ranged from .15 to .61 for Sample 1 and .07 to .60 for Sample 2.

Exploratory-Factor Analysis (Sample 1). Results of the exploratory-factor analysis (EFA) suggested the existence of three factors. The first factor (eigenvalue $=3.37$ ) was composed of six items with factor loadings ranging from .49 to .73. The second factor (eigenvalue $=1.18$ ) consisted of two items referring to physical activity during recess and physical education class, which had loadings of .41 and .59 , respectively. The item referring to physical activity at lunch did not load with either of the other two factors and stood alone as a single-item factor with an eigenvalue of 1.04. Despite the production of three factors by the EFA, the validity of a three-factor model is questionable because of the inconsistency with the literature $(7,21)$, the presence of a single-item factor, and low eigenvalue of the third factor. Furthermore, school policies in the districts from which the Study 1 sample was drawn did not allow physical activity during the lunch period, which suggests that the lunch item was not appropriate for the current sample. Therefore,

Table 1 Sample 1 and Sample 2 From Study 1, $M(S D)$

\begin{tabular}{lcc}
\hline & Sample 1 & Sample 2 \\
\hline Age (years) & $10.75(.50)$ & $10.73(.48)$ \\
PAQ-C ${ }_{\text {score }}{ }^{*}$ & $3.19(.64)$ & $3.22(.60)$ \\
Fasting glucose $(\mathrm{mg} / \mathrm{dL})$ & $86.98(11.99)$ & $86.24(7.89)$ \\
Percent body fat & $27.61(11.48)$ & $27.82(11.78)$ \\
Body-mass index $\left(\mathrm{kg} / \mathrm{m}^{2}\right)$ & $22.34(5.65)$ & $22.26(5.53)$ \\
Step test score & $65.85(13.79)$ & $66.22(15.72)$ \\
Cronbach's $\alpha$ for PAQ-C & .74 & .70 \\
\hline
\end{tabular}

Note. PAQ-C score is the average of the nine items for Study 1. 
another exploratory-factor analysis was conducted with Sample 1 after we omitted the lunch item.

Results of the EFA after the lunch item was omitted again suggested the existence of two factors. The first factor (eigenvalue $=3.34$ ) was composed of six items with factor loadings ranging from .51 to .74. The second factor (eigenvalue $=1.19$ ) consisted of two items referring to physical activity during recess and physical education class, which had loadings of .47 and .48 , respectively. Based on these results, a two-factor structure, omitting the lunch item, was tested utilizing CFA with Sample 2.

Confirmatory-Factor Analysis (Sample 2). Results of the CFA indicated that a two-factor model was an acceptable fit for the data, $\chi^{2}(55)=246.11$, RMSEA $<.01$ $(90 \% \mathrm{CI}=.00-.04), \mathrm{CFI}=1.00, \mathrm{NNFI}=1.00$. Parameter estimates (standardized coefficients) ranged from .40 to .75 for all items in the model. The $t$ values for all items in the scale were greater than 6.18 , indicating that each item was adequately measured.

Concurrent-Construct Validity (Sample 2). PAQ-C score based on Factor 1 was significantly correlated only with percentage body fat $(r=-.10, p<.05)$. The correlation between PAQ-C score and CVF approached significance $(r=.08, p<$ .08 ) in Hispanic children but was not significantly correlated with BMI or fasting glucose levels. Correlations for children of other ethnicities were not run because of insufficient numbers of participants in each group.

\section{Study 2}

\section{Method}

Participants and Procedures. Participants $(N=617)$ for this study were in an ongoing clinical trial in a large school district consisting of a mixture of urban and suburban schools in the southeastern United States. Parental report of racial origin indicated 61.3\% African American, 30.5\% European American, 1.3\% Hispanic, 0.5\% Asian American, 0.4\% Native American, $4.5 \%$ indicating "multiracial," and $1.5 \%$ not indicating race. Sixty-eight percent were eligible for free or reduced school lunch (school-district data). For the purpose of racial comparisons, only European American and African American participants were included in the analysis. This resulted in 566 participants, but 152 indicated that they had been sick in the previous week or provided incomplete data. Therefore, the present study used data from 414 third-grade students (mean age $=8.7 \pm .57$; 146 European American, 268 African American; 213 male, 201 female) for whom complete data were available.

The measures used in the present study were part of a larger battery of testing conducted for the Medical College of Georgia (MCG) FitKid Project (43). The test battery consisted of numerous physical and physiological measurements and is described elsewhere (43). All procedures were approved by the Human Assurance Committee at the Medical College of Georgia. The informed written consent of the parent or legal guardian of each participant was obtained, along with the assent of the participants, before data collection. On the testing day, students were gathered in a classroom where the questionnaires were administered by research assistants. 
All students were given uniform instructions, and all questions were read aloud. A minimum of a 1:10 research-assistant:participant ratio was maintained throughout testing. On completion of the questionnaires, students were taken to the MCG mobile testing laboratory in groups of 4 for anthropometric measures and fitness testing. All analyses were conducted on data collected in fall 2003.

Measures. Physical activity was assessed using the PAQ-C (7). Because this sample was younger than the previous sample, a reading expert from the participants' school district was employed to determine the appropriateness of the items. Based on experience acquired during Study 1, the recommendations of the expert, and the results of focus-group testing, the PAQ-C was slightly modified for Study 2 to make the activity questions in PAQ-C more appropriate for the age of the participants and the southern geographic locale. For example, the term aerobic exercise was changed to group exercise, and cross-country skiing was changed to water or snow skiing. Based on the results of the previous study and the policies of the school district from which the sample was drawn (they do not allow activity during lunch), Item 4, regarding activity at lunch, was omitted from all analyses and scale score calculations.

The Physical Activity Enjoyment Scale (PACES) (25) was used to measure enjoyment of physical activity. The PACES is a 16-item scale that employs a 5 -point Likert-type scale $(1=$ disagree a lot to $5=$ agree $a$ lot $)$ to discern participants' agreement with positive and negative statements with the stem "When I am physically active ..." such as "I enjoy it" or "It feels good." A score for enjoyment is computed by calculating the average of the 16 items. A score is not computed for participants with missing responses for more than two items. The PACES was found to be internally reliable in the present sample $(\alpha=.87)$.

The Self-Perception Profile for Children (SPPC) is a 36-item scale that measures children's domain-specific perceptions of their competence and assesses their global perception of their self-worth (16). The SPPC contains five 6-item subscales encompassing multiple facets of the self. For Study 2 only global self-worth, athletic competence, and physical appearance were administered to the participants. Acceptable internal consistency has been reported for children in third to fifth grade, with Cronbach's alpha of .78 for global self-worth, .80 for athletic competence, and .80 for physical appearance (17). For the present study, Cronbach's alpha was considerably lower for each subscale (global self-worth $\alpha=.64$, athletic competence $\alpha=.61$, and physical appearance $\alpha=.66$ ) than those reported previously.

The Task and Ego Orientation in Sport Questionnaire (TEOSQ) was used to measure individual dispositional differences in goal orientation in sport and physical activity (10). The TEOSQ is a 13-item scale that asks participants to respond to the stem "I feel most successful in sport when ..." and indicate their accordance with task-oriented (e.g., "when I do my very best") and ego-oriented (e.g., "when others can't do as well as me") phrases indicating subjective success. For the present study, the stem was slightly expanded to ". . . sport and physical activity. ..." Agreement was indicated using a 5-point Likert-type scale $(1=$ disagree a lot to 5 $=$ agree a lot ). The TEOSQ has been found to be internally consistent in children ages 9-12 (Cronbach's $\alpha$ ranging from .52 to .86 for the task subscale and from .59 to .86 for the ego subscale) (9). The TEOSQ subscales were also found to be internally reliable in the present sample (task $\alpha=.90$ and ego $\alpha=.81$ ). 
CVF was measured with a step test as in Study 1. In response to pilot data suggesting that the testing protocol was too taxing for the younger participants in Study 2, CVF was assessed using the YMCA step-test protocol, which is a shorter, less demanding submaximal field-based test (14). The step-test protocol required each participant to step up and down on a $30-\mathrm{cm}(12-\mathrm{in}$.) bench at a rate of 24 steps/min for $3 \mathrm{~min}$. Heart rate was recorded using a Polar heart-rate monitor (Model \#610i, Polar Electro, Finland) immediately on cessation of the test $\left(t_{0}\right)$ and at $1\left(t_{1}\right)$ and $2 \mathrm{~min}\left(t_{2}\right)$ posttest. A CVF score is calculated as described in Study 1.

Systolic blood pressure and diastolic blood pressure were measured with an automated blood-pressure device (Dinamap, Critikon, Inc., Tampa, FL). Measurements were made after a 5-min seated rest. While the participant remained seated, five readings were taken at 1-min intervals, and the last three were averaged to produce the systolic and diastolic blood-pressure values.

Weight and height were recorded using a digital scale (841 Bella Digital Scale, Seca) and stadiometer (Road Rod, Modern Measurement Devices), respectively. Both height (accurate to $0.1 \mathrm{~cm}$ ) and weight (accurate to $0.1 \mathrm{~kg}$ ) were recorded twice and averaged to produce the final value. Body-mass index (BMI) was calculated, and students were measured standing in their school clothes, as in Study 1.

\section{Statistical Analysis}

An ANOVA was conducted to determine any race or sex differences on the PAQ-C while controlling for the effect of age. To determine the concurrent validity of the PAQ-C, Pearson product-moment correlations were calculated for all measured variables. SPSS 11.05 was used to produce these statistics. CFA was conducted to test the two-factor model of the PAQ-C as performed in Study 1. In addition, the equality of the factor structure was examined across racial groups.

\section{Results}

Descriptive Statistics. Descriptive statistics for Study 2 can be found in Table 2. After adjusting for age, there was no significant sex effect or interaction effect between sex and age or race and age for the composite PAQ-C score. The internalconsistency coefficient (Cronbach's $\alpha$ ) for the PAQ-C was .64 for all participants, .75 for European American children, .56 for African American children, .66 for males, and .62 for females. The corrected item-total correlations ranged from .17 to .60 for European American children, .04 to .47 for African American children, .11 to .54 for males, and .10 to .46 for females.

Concurrent-Construct Validity. Table 3 shows the correlation coefficients between the physical measures and the PAQ-C score based on Factor 1 by race. PAQ-C score was significantly correlated with systolic blood pressure $(r=-.17, p$ $<.05), \mathrm{CVF}(r=.30, p<.01)$, and BMI $(r=-.16, p=.05)$ in European American children, but it was not significantly correlated with any physical measure in African American children. PAQ-C score was not significantly correlated with diastolic blood pressure for either race. 
Table 2 Study 2 by Race, $M(S D)$

\begin{tabular}{lcc}
\hline & $\begin{array}{c}\text { European } \\
\text { Americans }\end{array}$ & $\begin{array}{c}\text { African } \\
\text { Americans }\end{array}$ \\
\hline Age & $8.72(.53)$ & $8.75(.58)$ \\
PAQ-C $_{\text {score }}$ & $3.36(.80)$ & $3.37(.69)$ \\
Systolic blood pressure $(\mathrm{mmHg})$ & $109.46(9.71)$ & $111.23(8.49)$ \\
Diastolic blood pressure $(\mathrm{mmHg})$ & $64.80(4.97)^{*}$ & $66.51(5.07)$ \\
Step test score & $47.73(7.23)^{*}$ & $51.13(8.17)$ \\
Body-mass index $\left(\mathrm{kg} / \mathrm{m}^{2}\right)$ & $19.19(4.31)$ & $18.99(4.14)$ \\
Cronbach's $\alpha$ for PAQ-C & .75 & .56 \\
\hline
\end{tabular}

Note. PAQ- $\mathrm{C}_{\text {score }}=$ the average of the eight items, excluding the lunch item.

$* p<.01$.

Table 3 Correlations Between Physical Measures and PAQ-C Score by Race for Study 2

\begin{tabular}{|c|c|c|c|c|}
\hline & SBP & DBP & CVF & BMI \\
\hline \multicolumn{5}{|l|}{ European American } \\
\hline $\begin{array}{l}\text { PAQ- } \mathrm{C}_{\text {score }} \\
\text { systolic blood pressure (SBP) } \\
\text { diastolic blood pressure (DBP) } \\
\text { cardiovascular fitness (CVF) } \\
\text { body-mass index (BMI) }\end{array}$ & $-.17 *$ & $\begin{array}{l}.03 \\
.51 * *\end{array}$ & $\begin{array}{r}.30 * * \\
-.42 * * \\
-.22 * *\end{array}$ & $\begin{array}{r}-.16^{*} \\
.54^{* *} \\
.16^{*} \\
-.54 * *\end{array}$ \\
\hline \multicolumn{5}{|l|}{ African American } \\
\hline $\begin{array}{l}\text { PAQ-C } \\
\text { systore } \\
\text { diastolic BP } \\
\text { cardiovascular fitness } \\
\text { body-mass index }\end{array}$ & .03 & $\begin{array}{l}-.05 \\
.37 * *\end{array}$ & $\begin{array}{c}.02 \\
-.20 * * \\
-.17 *\end{array}$ & $\begin{array}{l}.01 \\
.32 * * \\
.02 \\
-.47 * *\end{array}$ \\
\hline
\end{tabular}

PAQ-C score was significantly correlated with athletic competence in African American children $(r=.14, p<.05)$ and European American children $(r=.14$, $p<.01)$. The correlation between PAQ-C score and enjoyment of physical activity approached significance in European American $(r=.16, p=.06)$ and African American $(r=.11, p=.07)$ children. PAQ-C score was not significantly correlated with task orientation, ego orientation, global self-worth, or physical appearance for either race.

Confirmatory-Factor Analysis. Results of the CFA indicated that the two-factor model developed in Study 1 was an acceptable fit for the data, $\chi^{2}(19)=65.71$, 
RMSEA $=.05(90 \% \mathrm{CI}=.03-.07), \mathrm{CFI}=.98, \mathrm{NNFI}=.96$. Parameter estimates (standardized coefficients) ranged from .26 to .72 for all items. The $t$ values for all items in the scale were greater than 2.29 , indicating that each item was adequately measured. The second analysis performed for African American and European American children to test the equality of the factor structure across race resulted in an unstable solution, $\chi^{2}(55)=303.24$, RMSEA $=.00(90 \% \mathrm{CI}=.00-.00)$, CFI $=1.00, \mathrm{NNFI}=1.02)$, possibly because of the relatively low number of European American participants $(n=146)$, and so it was not appropriate for interpretation.

\section{Discussion}

Study 1 sought to determine the factor structure of the PAQ-C in a predominantly Hispanic population of older children. Exploratory-factor analysis performed on Sample 1 produced a three-factor model, with one factor loaded on by six of the items and the other two factors with two and one item, respectively. After a retrospective examination of school policies for the schools from which participants were sampled, which require students to sit at their respective tables after eating, we reran these analyses with the omission of the item asking "In the last 7 days, what did you normally do at lunch (besides eating lunch)?" After we reran the EFA, we determined that a two-factor structure was most appropriate. The CFA in Sample 2 of Study 1 supported the results of the EFA with Sample 1, which suggested a two-factor solution.

The CFA performed in Study 2, which involved a mixture of African American and European American children, confirmed that a two-factor structure was indeed stable across samples. Unfortunately, because of sample size and model complexity, a factor-invariance model between races was uninterruptible. Study 2 also sought to determine the construct and concurrent validity of the PAQ-C. With respect to the construct validity of the PAQ-C, there appeared to be some consistent ethnic differences that could not be explained by sex, geographic location, or age. In Study 2, there were stronger relationships between PAQ-C score, fitness, and fatness for European American children than those observed in African American (Study 2) or Hispanic children (Study 1), although fatness was significantly correlated with PAQ-C score in Hispanic children. For European American children, the correlation of the PAQ-C score was .30 with CVF and -.16 with BMI in Study 2. It is interesting that no correlations greater than .08 were observed for the PAQ-C and any physical measure in the African American or Hispanic children. Although compelling, these differences are difficult to explain in the current study. One possible explanation for the racial differences might be differences in reading comprehension. The schools with a higher percentage of students eligible for free and reduced lunch (as a marker of socioeconomic status) tended to have a higher percentage of African American students. In turn, the schools with a higher percentage of students eligible for free and reduced lunch tended to do more poorly on the standardized reading-comprehension tests (42). Unfortunately, the aggregate nature of the present data prevents us from testing this hypothesis explicitly.

Regarding the concurrent validity of the PAQ-C in Study 2, the PAQ-C was significantly correlated with athletic competence in European American $(r=.24)$ 
and African American $(r=.14)$ children, which is far lower than previously reported in Canadian children $(r=.48)$ (21). The magnitude across the racial categories was similar in Study 2, however, suggesting that racial differences were not responsible for the smaller correlations. On the contrary, it would appear that the differences were most likely a result of reading-comprehension differences related to age, evidenced by the lower internal consistency for the SPPC subscales and the PAQ-C in Study 2 than those previously published $(7,17)$.

The findings of the two studies reported here suggest that the PAQ-C requires more work before it becomes an appropriate measure of young children from diverse racial groups. The present findings highlight many of the complications with assessing physical activity in children using self-report measures. With openended questionnaires, recall becomes a question in elementary-school-age children. With more structured questionnaires, such as the PAQ-C, the choice of questions relative to environmental factors (e.g., in school, at home) and psychometric concerns can become problematic. In the present study, this can be observed by examining the separate factors resulting from the EFA in Study 1, which appear to be tapping into the variance resulting from the setting in which the physical activity is conducted. The items loading on Factor 1 describe physical activity almost exclusively conducted away from school (e.g., "right after school," "on how many evenings"). The other items that load on Factor 1 are much more general in nature, asking about activity in "free time" or activity for "each day last week." This is in contrast to the two items loading on Factor 2, which ask about activity in "recess" and "physical education classes." These factors would appear to be sensitive to the location of the activity, which, if validated as such, would provide important information. Currently, the PAQ-C includes an item for activity at lunch that would be assumed to load with the other "in-school" items. Because of the documented school policies that do not allow activity during lunch for both populations in the present study, this was not the result of the EFA. This inconsistency should be considered when deciding to include this item, especially in studies that sample from different schools.

There are a number of limitations that should be considered when interpreting these reported findings. First, the data from the two studies presented in this article come from larger randomized controlled trials that were not designed specifically for the validation of the PAQ-C. The nature of data collection in a field setting can interject random error into measurement, especially in data that are collected by a questionnaire, which might have suppressed the reliability of both the PAQ-C and the other questionnaires used in the study. Considerable effort was made by the research staff to minimize this source of error, but it cannot be guaranteed that the poor psychometric performance of the PAQ-C relative to previous studies was not because of this additional source of error. Similar concerns relate to the measurement of fasting glucose and body composition in Study 1. The inability to stringently control for hydration or fasting status undoubtedly affected the results, which would suppress any significant findings. Previous published findings using these measures of fasting glucose and body composition in this sample have yielded significant results, however, suggesting that the physiological data are valid.

In conclusion, these findings would suggest that the PAQ-C requires additional development to be a useful measure of physical activity in American children from 
diverse ethnic populations. The findings of the two studies presented here provide additional information to the initial validation studies of the PAQ-C, which will be useful for future studies seeking to develop easily administered questionnaires to assess physical activity in children.

\section{Acknowledgments}

This research was supported by grants from the National Institute of Diabetes and Digestive and Kidney Diseases (R01DK063391 and RO1DK59213). The authors would like to thank the San Antonio School Board and the Richmond County School Board for their assistance. We would also like to thank Ms. Marlo Cavnar and Ms. Janet Thornburg for their assistance in data collection and Ms. Deanna Owen for her careful proofreading and editing.

Correspondence concerning this article should be addressed to Justin B. Moore, PhD, Master of Public Health Program, Division of Community Health and Preventitive Medicine, The Brody School of Medicine at East Carolina University, 1709 West 6th Street, Greenville, NC 27834, (252) 744-4039, MOOREJ@ecu.edu

\section{References}

1. American Diabetes Association. Screening for Type 2 diabetes. Diabetes Care. 27:11S14S, 2004.

2. Barr, S., and H. McKay. Nutrition, exercise, and bone status in youth. Int. J. Sport Nutr. 8:124-142, 1998.

3. Bild, D., D. Jacobs, S. Sidney, W. Haskell, N. Anderssen, and A. Oberman. Physical activity in young Black and White women: the Cardia Study. Ann. Epidemiol. 3:636644, 1993.

4. Browne, M.W., and R. Crudeck. Alternate ways of assessing model fit. In: Testing Structural Equation Models. K.A. Bollen and J.S. Long (Eds.). Thousand Oaks, CA: Sage Publications, 1993.

5. Caspersen, C., M. Pereira, and K. Curran. Changes in physical activity patterns in the United States, by sex and cross-sectional age. Med. Sci. Sports Exerc. 32:1601-1609, 2000.

6. Centers for Disease Control and Prevention. Youth Risk Behavior Surveillance-United States, 2003.Washington, DC: Centers for Disease Control and Prevention, 2004.

7. Crocker, P.R.E., D. Bailey, R. Faulkner, K.C. Kowalski, and R. McGrath. Measuring general levels of physical activity: preliminary evidence for the physical activity questionnaire for older children. Med. Sci. Sports Exerc. 29:1344-1349, 1997.

8. Crocker, P.R.E., R.C. Eklund, and K.C. Kowalski. Children's physical activity and physical self-perceptions. J. Sports Sci. 18:383-394, 2000.

9. Duda, J.L. Advances in Sport and Exercise Psychology Measurement. 2nd ed. Morgantown, WV: Fitness Information Technology, 2000.

10. Duda, J.L., and J.G. Nicholls. Dimensions of achievement motivation in schoolwork and sport. J. Educ. Psychol. 84:290-299, 1992.

11. Ekelund, U., J. Aman, A. Yngve, C. Renman, K. Westerterp, and M. Sjostrom. Physical activity but not energy expenditure is reduced in obese adolescents: a case-control study. Am. J. Clin. Nutr. 76:935-941, 2002.

12. Ellis, K.J. Selected body composition methods can be used in field studies. J. Nutr. 131:1589S-1595S, 2001.

13. Girouard, J., J. Forest, J. Masse, et al. Multicenter evaluation of the Glucometer Elite XL meter, an instrument specifically designed for use with neonates. Diabetes Care. 23:1149-1153, 2000. 
14. Golding, L.A. YMCA Fitness Testing and Assessment Manual. 4th ed. Champaign, IL: Human Kinetics, 2000.

15. Gutin, B., and S. Owens. Role of exercise intervention in improving body fat distribution and risk profile in children. Am. J. Hum. Biol. 11:237-247, 1999.

16. Harter, S. The perceived competence scale for children. Child Dev. 53:87-97, 1982.

17. Harter, S. Manual for the Self-Perception Profile for Children. Denver, CO: University of Denver, 1985.

18. Joreskog, K.G., and D. Sorbom. LISREL. Chicago, IL: Scientific Software International, 2003.

19. Kimm, S.Y.S., N.W. Glynn, A.M. Kriska, et al. Decline in physical activity in Black girls and White girls during adolescence. N. Engl. J. Med. 347:709-715, 2002.

20. Kline, R.B. Principles and practice of structural equation modeling. In: D.A. Kenny (Ed.), Methodology in the Social Sciences. New York, NY: Guilford Press, 1998:127131.

21. Kowalski, K.C., P.R.E. Crocker, and R.A. Faulkner. Validation of the physical activity questionnaire for older children. Pediatr. Exerc. Sci. 9:174-186, 1997.

22. MacCallum, R.C., and J.T. Austin. Applications of structural equation modeling in psychological research. Ann. Rev. Psychol. 51:201-226, 2000.

23. Marsh, H.W., F. Asci, and I.M. Thomas. Multitrait-multimethod analyses of two physical self-concept instruments: a cross-cultural perspective. J. Sport Exerc. Psychol. 24:99$119,2002$.

24. Morrison, J., B. Barton, F. Biro, S. Daniels, and D. Sprecher. Overweight, fat patterning, and cardiovascular disease risk factors in Black and White boys. J. Pediatr. 135:451457, 1999.

25. Motl, R., R.K. Dishman, R. Saunders, M. Dowda, G. Felton, and R.R. Pate. Measuring enjoyment of physical activity in adolescent girls. Am. J. Prev. Med. 21:110-117, 2001.

26. Motl, R.W., A.S. Birnbaum, M.Y. Kubik, and R.K. Dishman. Naturally occurring changes in physical activity are inversely related to depressive symptoms during early adolescence. Psychosom. Med. 66:336-342, 2004.

27. National Association for Sport and Physical Education. Physical Activity for Children: A Statement of Guidelines for Children Ages 5-12. 2nd ed. Reston, VA: National Association for Sport and Physical Education, an Association of the American Alliance for Health, Physical Education, Recreation and Dance, 2004.

28. Rowlands, A.V., R.G. Eston, and D.K. Ingledew. Relationship between activity levels, aerobic fitness, and body fat in 8- to 10-yr-old children. J. Appl. Physiol. 86:1428-1435, 1999.

29. Sallis, J.F. Epidemiology of physical activity and fitness in children and adolescents. Crit. Rev. Food Sci. Nutr. 33:403-408, 1993.

30. Sallis, J.F., and K. Patrick. Physical activity guidelines for adolescents: consensus statement. Pediatr. Exerc. Sci. 6:302-314, 1994.

31. Sallis, J.F., and B.E. Saelens. Assessment of physical activity by self-report: status, limitations, and future directions. Res. Q. Exerc. Sport. 71:1, 2000.

32. Sallis, J.F., J.M. Zakarian, M.F. Hovell, and C.R. Hofstetter. Ethnic, socioeconomic, and sex differences in physical activity among adolescents. J. Clin. Epidemiol. 49:125-134, 1996.

33. Schmidt, G., J. Walkuski, and D. Stensel. The Singapore youth coronary risk and physical activity study. Med. Sci. Sports Exerc. 30:105-113, 1998.

34. Shephard, R.J., and A. Vuillemin. Limits to the measurement of habitual physical activity by questionnaires. Br. J. Sports Med. 37:197-206, 2003.

35. Strauss, R.S., D. Rodzilsky, G. Burack, and M. Colin. Psychosocial correlates of physical activity in healthy children. Arch. Pediatr. Adolesc. Med. 155:897-902, 2001 
36. Suter, E., and M. Hawes. Relationship of physical activity, body fat, diet, and blood lipid profile in youths 10-15 yr. Med. Sci. Sports Exerc. 25:748-54, 1993.

37. Tomson, L.M., R.P. Pangrazi, G. Friedman, and N. Hutchison. Childhood depressive symptoms, physical activity and health related fitness. J. Sport Exerc. Psychol. 25:419439, 2003.

38. Tremblay, M.S., J.W. Inman, and J.D. Willms. The relationship between physical activity, self-esteem, and academic achievement in 12-year-old children. Pediatr. Exerc. Sci. 12:312-323, 2000.

39. Trevino, R.P., Z. Yin, A. Hernandez, D.E. Hale, O.A. Garcia, and C. Mobley. Impact of the Bienestar school-based diabetes mellitus prevention program on fasting capillary glucose levels: a randomized controlled trial. Arch. Pediatr. Adolesc. Med. 158:911917, 2004.

40. Washburn, R.A., G. Kline, D.T. Lackland, and F.C. Wheeler. Leisure time physical activity: are there Black/White differences? Prev. Med. 21:127-35, 1992.

41. Welten, D., H. Kemper, G. Post, et al. Weight-bearing activity during youth is a more important factor for peak bone mass than calcium intake. J. Bone Miner. Res. 9:10891096, 1994.

42. Yin, Z., B. Gutin, M.H. Johnson, et al. An environmental approach to obesity prevention in children: MCG Fitkid project year 1 results. Obes. Res. 13:2153-2161, 2005.

43. Yin, Z., J. Hanes, Jr., J.B. Moore, P. Humbles, P. Barbeau, and B. Gutin. An after-school physical activity program for obesity prevention in children: the Medical College of Georgia Fitkid project. Eval. Health Prof. 28:67-89, 2005. 